\title{
Motion Tracking Algorithms for Inertial Measurement
}

\author{
Javier Torres \\ Tyndall National \\ Institute \\ University College \\ Cork \\ Ireland \\ 00353214904376
}

javier.torres@tyndall.ie

\author{
Brendan O'Flynn \\ Tyndall National \\ Institute \\ University College \\ Cork \\ Ireland \\ 00353214904088
}

boflynn@tyndall.ie

\author{
Philip Angove \\ Tyndall National \\ Institute \\ University College \\ Cork \\ Ireland \\ 00353214904401
}

pangove@tyndall.ie

\author{
Frank Murphy \\ Tyndall National \\ Institute \\ University College \\ Cork \\ Ireland \\ 00353214904088
}

fmurphy@tyndall.ie

\author{
Cian O' Mathuna \\ Tyndall National \\ Institute \\ University College \\ Cork \\ Ireland \\ 00353214904088
}

omathuna@tyndall.ie

\begin{abstract}
In this paper, we describe the development of the software algorithms required to interpret sensor data developed by a wearable miniaturized wireless inertial measurement unit (IMU) to enable tracking of movement
\end{abstract}

Traditionally, inertial tracking has involved the use of off the shelf motion sensors in the form of an inertial measurement unit, in combination with a GPS based receiver system for improved accuracy. Several immediate concerns are evident when a low cost, low power consumption, miniaturised solution is needed in applications such as animal tracking. GPS solutions have proven to be costly requiring an expensive satellite link \& entail power supply and size concerns when deployed on live animals. In particular applications, GPS coverage is not available for all application scenarios and alternative mechanisms for motion tracking are required. IMUs cannot be used in isolation for absolute position tracking, since an IMU calculates position utilising a square function of time $(t)$ where the error is proportional to the sampling time, any errors in the output of the sensors are therefore also multiplied by $\mathrm{t}^{2}$. This typically leads to large positional errors in operation: These issues can potentially be addressed by using only a low cost modular IMU solution to enable the mapping of movement if appropriate algorithms are implemented

The goal of this paper is to present a mathematical algorithm that enables an inertial -based tracking system to be realized. This algorithm could then be used with GPS (GPS is commonly used but there are other methods like triangulation) along with a Kalman Filter algorithm providing an accurate 3Dimensional tracking system.

\section{Categories and Subject Descriptors}

D.2.4 Algorithms, B.4.1 Data Communications Devices, E.1 Data Structures, F.2.1 Numerical Algorithms \& Problems

\section{General Terms}

Algorithms, Measurement, Design, Reliability, Experimentation

\section{Keywords}

Wireless Inertial Measurement Unit (WIMU). Kalman Filter, Motion Tracking, algorithms.

\section{INTRODUCTION}

Autonomous position mapping can be realized using a miniaturized wireless IMU with 3-axis sensor outputs indicating acceleration, angular velocity \& magnetic field. Using this information as inputs to a system of equations representing IMU motion, the resultant positional displacement, angular rotation \& compass orientation relative to the earth's magnetic field, in all 3axes can be realized.

This positional data could for instance be utilised with GIS mapping software to see the movement of the body either in real time following data download, or interact directly with the environment [1], depending on the application.

The inherent drift common in all IMU sensors can be compensated for using an additional system as appropriate e.g. an RFID system to give a zero (origin) datum point correction, or another application specific zeroing mechanism (GPS, triangulation etc.). Furthermore filtering algorithms such as Kalman filtering [2] [3] [4] or digital filtering of the sensor outputs can be enabled for improved accuracy.

\section{WIMU Hardware Development}

The 25mm WIMU, has been described previously [5], and is a 6 DOF IMU module, which is made up of an array of inertial sensors coupled with a high resolution analog to digital converter (ADC). The $25 \mathrm{~mm}$ WIMU module utilises the wireless communication capabilities of the $25 \mathrm{~mm}$ wireless node [6] [7] to realise a fully autonomous WIMU module.

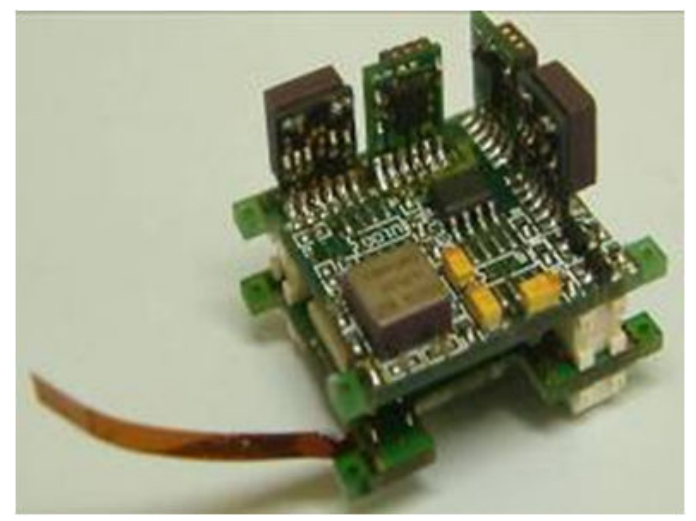

Figure 1. Tyndall's 25mm WIMU Module
Permission to make digital or hard copies of all or part of this work for personal or classroom use is granted without fee provided that copies are not made or distributed for profit or commercial advantage and that copies bear this notice and the full citation on the first page. To copy otherwise, to republish, to post on servers or to redistribute to lists, requires prior specific permission and/or a fee. BODYNETS 2007, June 11-13, Florence, Italy Copyright () 2007 ICST 978-963-06-2193-9 DOI 10.4108 /bodynets.2007.170 
The wireless node has an integrated ATMEL ATMega128 [8] microcontroller for the development of customised embedded protocol algorithms for the networking of the modules. This feature coupled with the $2.4 \mathrm{GHz}$ transceiver, RF Nordic nRF2401 [9], produces a very powerful customisable wireless node. Alternative implementations enable the Zigbee (IEEE 802.15.4) communications in the $25 \mathrm{~mm}$ form factor if required. The $25 \mathrm{~mm}$ WIMU can be seen in Figure 1. The inertial sensor array consists of three single axis gyroscopes, ADXRS150 [10] from Analog Devices, two dual axis accelerometers, ADXL202 [11] from Analog Devices, and two dual axis magnetometers, HMC1052L [12] from Honeywell. The sensor array was designed with a novel 3D structure, which produces the 6 required degrees of freedom (DOF) necessary to determine position. The module also has a 12bit ADC chip, AD7490 [13] from Analog Devices, handling the data conversion. This chip has a Serial Peripheral Interface (SPI), which allows easy interfacing to the ATMEL microcontroller, as outlined in Figure 2.

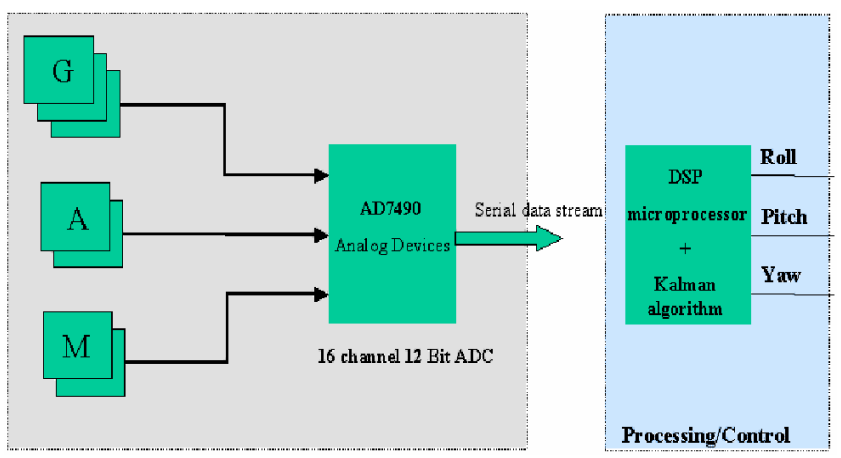

Figure 2. WIMU Block Diagram

The 3D structure was implemented using a motherboard/daughterboard configuration. Miniature slots were drilled in the motherboard to allow the daughterboard to be oriented at a $90^{\circ}$ angle with respect to the motherboard. The motherboard and daughterboard were designed so that their respective pads would register accurately and allow for a $90^{\circ}$ solder connection. The motherboard was configured so the sensors would make up the relevant array to generate the 6 degrees of freedom required for the module.

\section{3. $25 \mathrm{~mm}$ WIMU Characterisation}

The WIMU module has been developed using off the shelf components from suppliers such as Analog Devices and Honeywell. The performance of the module had to be tested for basic functionality and then for the generation of a set of system specifications. A number of experiments were designed and executed to carry out this characterisation. The system was connected to a PC wirelessly to log the information received from the WIMU for off board analysis. Each of the sensors was subject to conditions that excited the respective sensing properties (tilt, rotation, magnetic field) and the results were recorded and compared to manufacturers' specifications. The accelerometers were subject to tilt extremes to measure the max, min and zero offsets. The gyroscopes were excited using a calibrated rotating table while the magnetometers were excited using a Helmholtz coil set up. All of the data was recorded and analysed used a customised $\mathrm{C}++$ application.
The "Rock 'n' Roll" application was developed to provide a visual analysis of the sensor data being produced by the WIMU. This application allowed for the viewing of the sensor signals in real time and contained integrated calibration routines that allowed for the calculation of the resolution of each the sensors. The oscilloscope style interface of the 'Rock'n'Roll' application can be seen in Figure 3 .

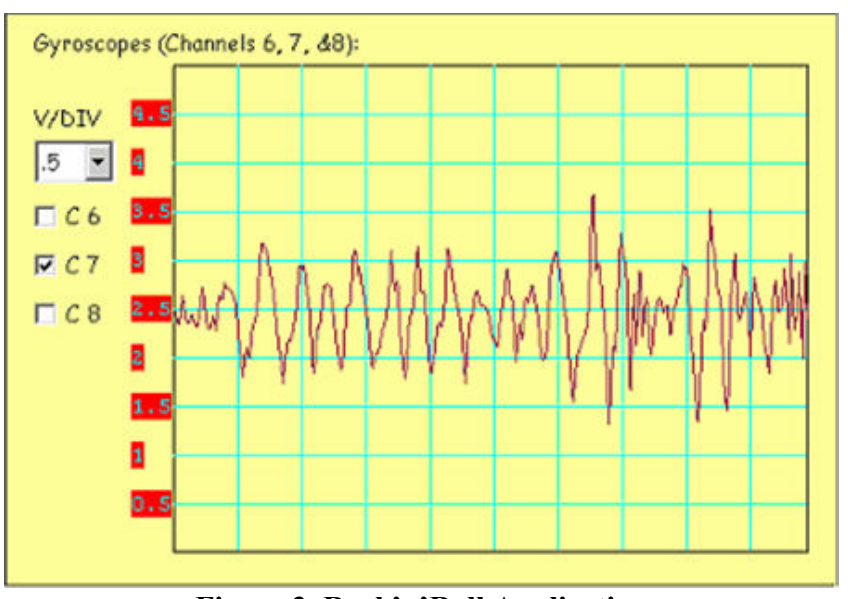

Figure 3. Rock'n'Roll Application

The Rock'n'Roll application also has a Kalman Filter module integrated to enable the fusion of the sensor signals and real time monitoring of the orientation of the module.

Using this application, the Gyroscopes, Accelerometers and Magnetometers were all tested and shown to be working within the expected parameters. The Gyroscopes recorded sensitivity was within $2 \%$ of the expected value. The Accelerometers all produced sensitivity factors within $5 \%$ of their expected specified values. The magnetometers were shown to have reduced sensitivity but still maintained sub degree resolution for high specification navigation applications. Reset of the magnetometers (under control of the microcontroller as required) increases dramatically the resolution of the magnetometers.

\subsection{Sensor Characterisation}

\subsubsection{Accelerometers}

As the name suggests, the IMU provides inertial acceleration measurements, therefore resulting measurements made by the accelerometers are a sum of effects due to linear acceleration, orientation of the platform with respect to the gravity vector, angular acceleration of the platform as it moves round a curve, the effects of the Coriolis force, and finally the centrifugal acceleration caused by the earth rotation around itself. Ignoring the last two acceleration components we can consider that the measurements made by the accelerometers are in fact a sum of effects due to linear acceleration, orientation of the platform with respect to the gravity vector and angular acceleration of the platform as it moves round a curve [14]. However accelerometers sensors will be mostly sensitive to the gravity vector element and therefore can also be used as a tilt sensor [15], their role when used as an inclinometer can be improved by using an appropriate low pass filter. 


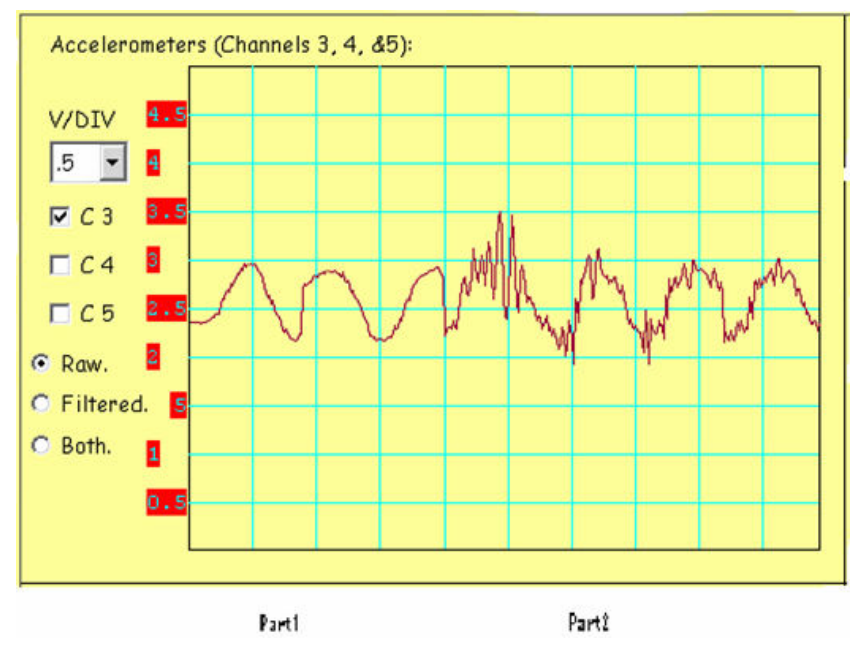

Figure 4: Signal output of the accelerometer with sensitive axis in the $U$ direction.

Figure 4 above graphically shows the signal output of the accelerometer which has its sensitive axis in the $U$ direction (see Figure 8). The first segment of the wave form shows the acceleration measured while we were changing the $U$ axis from a plane in which it's parallel to the earth's surface to a plane in which it points toward the earth's core and the sensor is experiencing a force of $1 \mathrm{~g}\left(9.81 \mathrm{~m} / \mathrm{s}^{2}\right)$. This is the acceleration due to tilt respect to gravity.

The second segment shows the acceleration measured with the same motion as the first section, but with simultaneous inclusion of dynamic acceleration.

\subsubsection{Gyroscopes}

Gyroscopes are angular velocity sensing devices. They provide the angular velocity around the three orthogonal axes. The first time integration of the angular velocity leads to the Euler angles that are necessary to describe the orientation of the IMU. The Euler angles are usually given in aeronautical term as Pitch, Roll and Yaw (See Figure 8), where: Pitch is the rotation around the lateral $(\mathrm{V})$ axis, Roll around the longitudinal (U) axis and Yaw around the perpendicular (W) one.

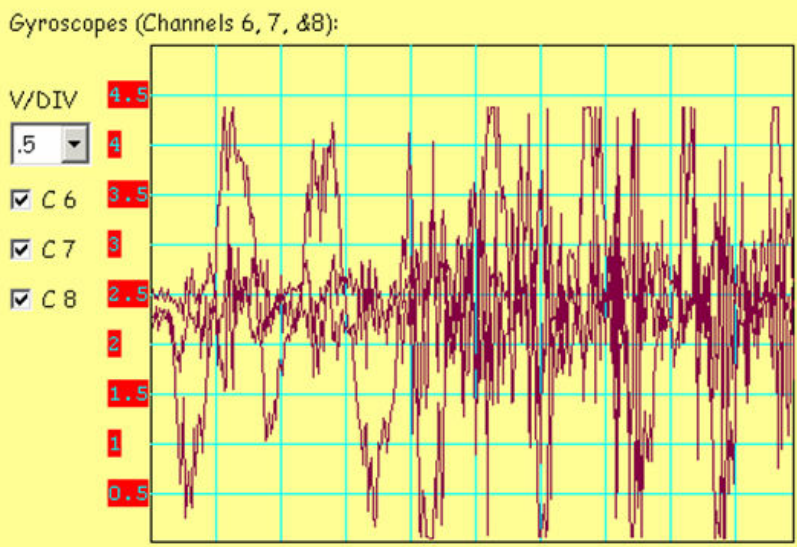

Figure 5. Gyroscopes' outputs

\subsubsection{Magnetometers}

The magnetometers integrated into the IMU system measure the earth magnetic field on three orthogonal axes. Figure 6 shows the magnetometer output in the West direction (see Figure 7) while we were changing its orientation from north to south.

The Earth's magnetic field intensity is about 0.5 to 0.6 Gauss, it is approximated with a dipole model and has a component parallel to the Earth's surface that always pointing toward magnetic north and is used to determine compass direction.

For establishing device orientation, the key components of the magnetometers' sensor data are a) parallel to the earth's surface and b) in the direction of the earth's magnetic north. These vectors can be used to estimate the direction cosine matrix (it will be called Orientation Matrix from now on) defined in section 4.1.

The end result of these calculations is an orientation estimation referenced with respect to the magnetic north, which differs from geographic north, by about 5 degrees in geographic locations found in Ireland. Geographic north is located at the earth's rotational axis and is referenced by the meridian lines found on maps. At different locations around the globe, magnetic and geographic north can differ by \pm 25 degrees. This difference is called the declination angle and can be determined from a lookup table based on the geographic location.

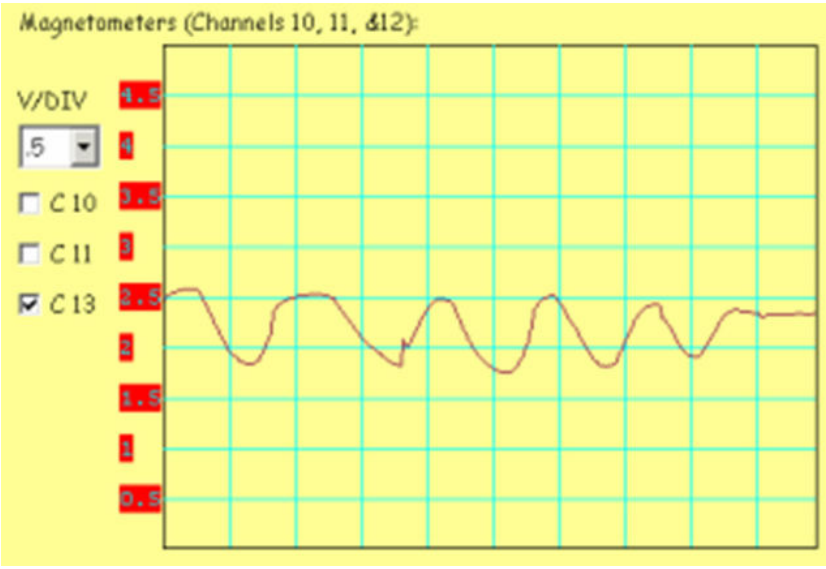

Figure 6: Magnetometers outputs

Generally it is possible to obtain the orientation of the IMU with high accuracy by fusing Gyroscope, Accelerometer, and Magnetometer signals via Kalman Filtering [16,17].

In this case, the accelerometers are used as a tilt sensor, under this approach is useful to use an appropriate low pass Filter that make the accelerometers signal more sensitive to gravity [18] and the magnetometers are used as a compass. 


\section{Inertial Systems Equations.}

\subsection{Frames}

Measurements made directly from the IMU are referenced with respect to the IMU sensors axes, which are fixed by the physical orientation of the sensors. These axes (Figure 7) are called the IMU-Fixed Frame and all the sensor outputs are referenced to this frame.
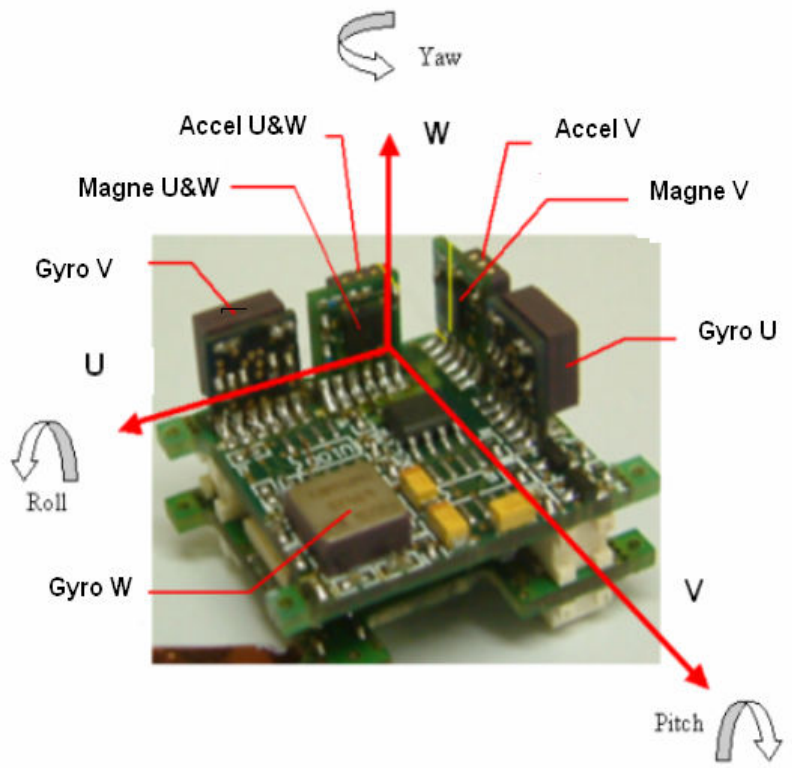

Figure 7. WIMU gyro, accelerometer and magnetometer layout and IMU-Fixed Frame.

To enable the tracking of positioning and orientation, we need to reference the data to a known origin (reference frame. To achieve this we use the Earth-Fixed Frame (Figure 8) that is defined with its X-axis parallel to the Earth's surface and pointing toward the Earth magnetic North, its Z-axis parallel to the gravity vector and therefore perpendicular to the Earth's surface and Yaxis will be the cross product between the $\mathrm{Z}$ and $\mathrm{X}$ axes.

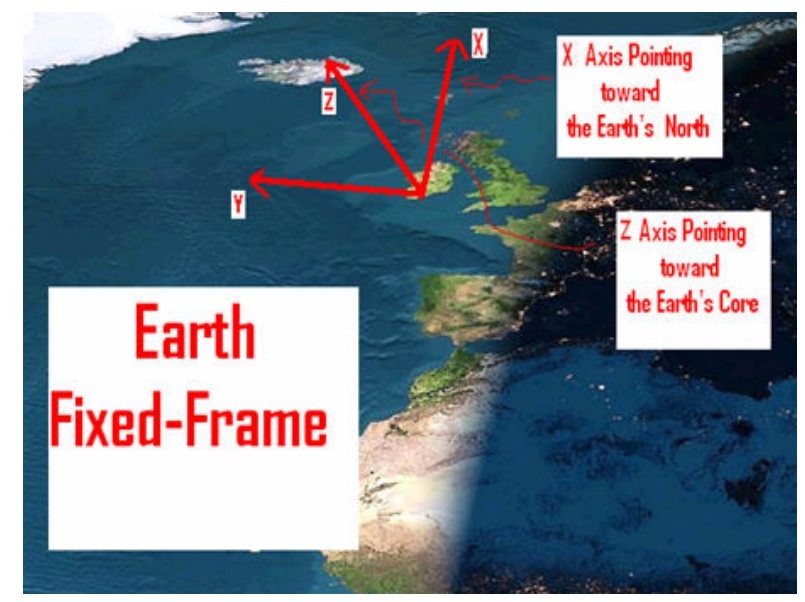

Figure 8. Earth Fixed Frame.

The Orientation Matrix is a $3 \times 3$ Matrix that transforms any vector given respect to the IMU fixed-Frame (and therefore given by the sensors outputs) into the known reference Earth-Fixed Frame.

\subsection{Algorithms Development for inertial (IMU)-based tracking system}

The 3-axis acceleration \& angular velocity sensor output values can be combined in a non-linear matrix equation to give both position \& orientation information.

The system can be visualized by using a fixed frame of reference for position measurement $(\mathrm{x}, \mathrm{y}, \mathrm{z})$ : The Earth-Fixed Frame and utilizing a moving non-inertial frame (u, v, w): IMUFixed Frame with its axes parallel to the IMU sensors axes (see Figures 7, 8 and 9)
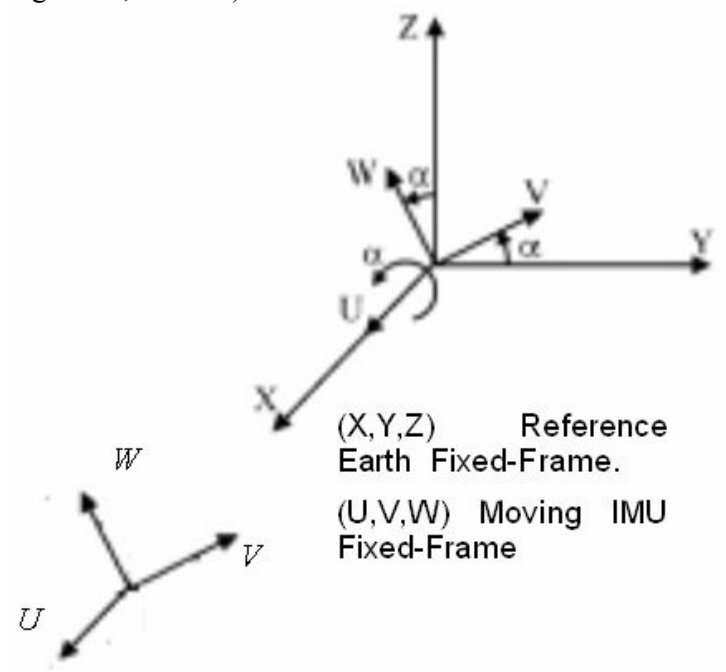

$(\mathrm{U}, \mathrm{V}, \mathrm{W})$ Moving IMU

Fixed-Frame

Figure 9. Moving and Reference frames

\subsubsection{Orientation Estimation Calculations}

The Orientation of the IMU will have two sources of estimation: gyroscopes outputs and the outputs from the accelerometers and magnetometers. Gyroscopes cannot be used in isolation for obtaining absolute Orientation because of the drift associated with their readings. Magnetometers \& accelerometers lead to orientation estimation with long-term stability but shortterm occasional inaccuracy due to the presence of ferromagnetic materials or other magnetic fields (different to the earth's magnetic field) and due to linear and rotational accelerations that disturb the orientation estimation.

Thus we can combine the advantage of the short-term precision of Gyroscopes and the long-term stability of Accelerometers and Magnetometers via a Kalman Filter.

\subsubsection{Orientation estimation from gyroscope outputs}

Every time step we are able to know the Euler angles $\alpha, \phi, \theta$, we can keep the order of rotation and follow it every time step as follows:

Definition of Angles

$$
\begin{aligned}
& \Delta \theta(k+1)=\Delta t \cdot \frac{\dot{\theta}(k+1)+\dot{\theta}(k)}{2} \quad(\text { Yaw }) \\
& \Delta \phi(k+1)=\Delta t \cdot \frac{\dot{\phi}(k+1)+\dot{\phi}(k)}{2} \quad \text { (Pitch) } \\
& \Delta \alpha(k+1)=\Delta t \cdot \frac{\dot{\alpha}(k+1)+\dot{\alpha}(k)}{2} \quad(\text { Roll })
\end{aligned}
$$


where:

$\Delta \theta$ denotes the incremental angle around the $\mathrm{W}$-axis, $\Delta \alpha$ denotes the incremental angle around the U-axis. $\Delta \phi$ denotes the incremental angle around the $\mathrm{V}$-axis. $\Delta t$ denotes the time step.

and $\mathrm{k}$ is the time index.

Note that the trapezium rule has been used in equations (2),(3)\&(4) as numerical integration method.

Given rotations matrix denoted as $\mathrm{R}$ around each particular axis [18]:

$$
\begin{aligned}
& R(W, \theta, k+1)=\left(\begin{array}{ccc}
\cos \Delta \theta(k+1) & -\sin \Delta \theta(k+1) & 0 \\
\sin \Delta \theta(k+1) & \cos \Delta \theta(k+1) & 0 \\
0 & 0 & 1
\end{array}\right) \\
& R(V, \phi, k+1)=\left(\begin{array}{ccc}
\cos \Delta \phi(k+1) & 0 & \sin \Delta \phi(k+1) \\
0 & 1 & 0 \\
-\sin \Delta \phi(k+1) & 0 & \cos \Delta \phi(k+1)
\end{array}\right) \\
& R(U, \alpha, k+1)=\left(\begin{array}{ccc}
1 & 0 & 0 \\
0 & \cos \Delta \alpha(k+1) & -\sin \Delta \alpha(k+1) \\
0 & \sin \Delta \alpha(k+1) & \cos \Delta \alpha(k+1)
\end{array}\right)
\end{aligned}
$$

and given general rotation matrix [18]:

$$
\text { Rotation }(k+1)=R(W, \theta, k+1) \cdot R(V, \phi, k+1) \cdot R(U, \alpha, k+1)
$$

We define an Orientation matrix as follows:

$$
\text { Orientation }(k+1)=\text { Rotation }(k+1) \cdot \text { Orientation }(k)
$$

The orientation matrix contains information about the orientation of the IMU and the order of rotation followed until this orientation is achieved. That means we can translate the coordinates of any vector from the IMU-Fixed Frame to the EarthFixed Frame using the Orientation Matrix.

\subsubsection{Orientation estimation from Accelerometers and Magnetometers outputs}

Reminding that the Orientation Matrix is a $3 \times 3$ Matrix that transforms any vector given respect to the IMU fixed-Frame (and therefore given by the sensors outputs) into the known reference Earth-Fixed Frame, so that the Orientation Matrix transforms coordinates from the IMU-fixed frame to the Earth-fixed frame and, equivalently, its inverse transforms coordinates from the Earth-fixed frame to the IMU-fixed frame.

Defining the Orientation Matrix inverse as:

$$
\text { Orientation }^{-1}=\left(\begin{array}{lll}
a_{11} & a_{12} & a_{13} \\
a_{21} & a_{22} & a_{23} \\
a_{31} & a_{32} & a_{33}
\end{array}\right)
$$

The magnetometer measurements provide data regarding the earth's magnetic field on three orthogonal axes. This magnetic field vector is used to directly calculate the first column of the inverse of Orientation Matrix.

Considering that the earth magnetic field vector, referenced to the Earth Fixed Frame, is parallel to the $\mathrm{X}$ axis and that $\mathrm{H}$ denotes the earth magnetic field magnitude in the region of interest, then, the Earth magnetic field coordinates in the Earth Fixed frame are $\left(\begin{array}{lll}H & 0 & 0\end{array}\right)$ and, if $\left(\begin{array}{lll}H_{u} & H_{v} & H_{w}\end{array}\right)$ is the magnetic field vector directly measured from the IMU outputs, and therefore representing its coordinates respect with the IMU Fixed frame, then:

$$
\left(\begin{array}{l}
H_{u} \\
H_{v} \\
H_{w}
\end{array}\right)=\left(\begin{array}{lll}
a_{11} & a_{12} & a_{13} \\
a_{21} & a_{22} & a_{23} \\
a_{31} & a_{32} & a_{33}
\end{array}\right) \cdot\left(\begin{array}{c}
H \\
0 \\
0
\end{array}\right)
$$

So the First column of the inverse of the Orientation Matrix is given as:

$$
\left(\begin{array}{l}
a_{11} \\
a_{21} \\
a_{31}
\end{array}\right)=\frac{1}{H} \cdot\left(\begin{array}{l}
H_{u} \\
H_{v} \\
H_{w}
\end{array}\right)
$$

As well as the magnetic vector, the accelerometer field vector is used to directly calculate the third column of the inverse of Orientation matrix.

Since the gravity vector, referenced to Earth fixed-frame, is parallel to the $\mathrm{Z}$ axis and $\mathrm{g}$ denotes the gravity acceleration magnitude, then, the gravity vector coordinates in the Earth Fixed frame are ( $\left.\begin{array}{lll}0 & 0 & g\end{array}\right)$ and, if ( $a_{\text {ugravity }} a_{\text {vgravity }}$ awgravity $)$ is the acceleration field vector directly measured from the IMU outputs, and therefore representing its coordinates respect with the IMU Fixed frame, then:

$$
\left(\begin{array}{l}
a_{\text {ugravity }} \\
a_{\text {vgravity }} \\
a_{\text {wgravity }}
\end{array}\right)=\left(\begin{array}{lll}
a_{11} & a_{12} & a_{13} \\
a_{21} & a_{22} & a_{23} \\
a_{31} & a_{32} & a_{33}
\end{array}\right) \cdot\left(\begin{array}{c}
0 \\
0 \\
g
\end{array}\right)
$$

So the Third column of the inverse of the Orientation Matrix is given as:

$$
\left(\begin{array}{l}
a_{13} \\
a_{23} \\
a_{33}
\end{array}\right)=\frac{1}{g} \cdot\left(\begin{array}{c}
a_{\text {ugravity }} \\
a_{\text {vgravity }} \\
a_{\text {wgravity }}
\end{array}\right)
$$

Finally the second column of the inverse of the orientation matrix will be directly calculated from the cross product between the third and first columns. 


\subsubsection{System equation for tracking position}

The general algorithm used to obtain position is presented in flow-chart in Figure 10.

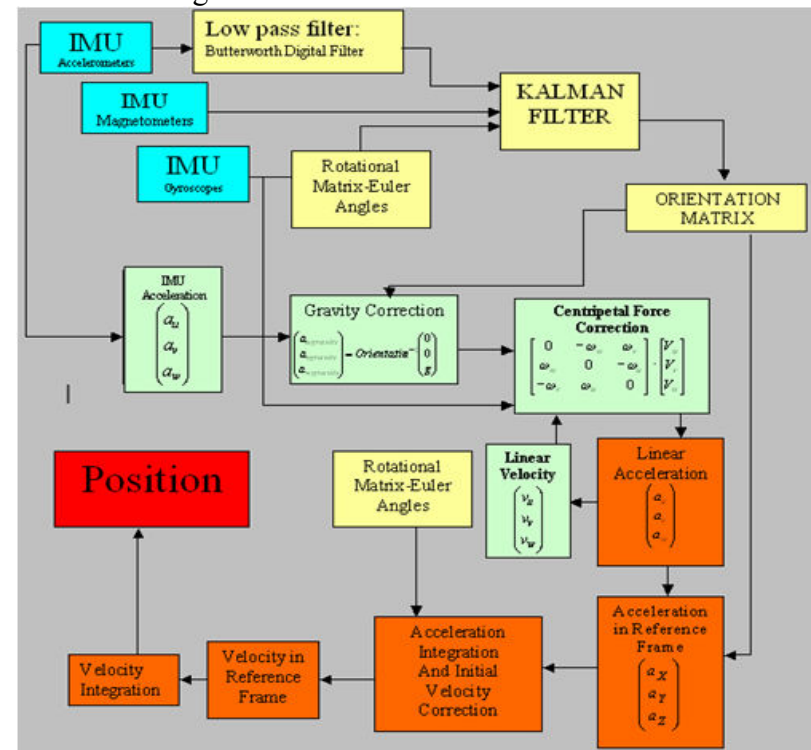

Figure 10. Inertial tracking algorithm flow chart algorithm:

It can be seen that three different elements comprise the

(a) The top element, which corresponds to the Orientation estimation.

(b) The middle element, which corresponds to the linear acceleration extraction.

(c) The button element, which corresponds to the system equation for obtaining position

The system equations for tracking position are then summarized in the following state equation

$X(k+1)=A \cdot[\operatorname{Rot}(k+1) \cdot X(k)]+B \cdot\left[\operatorname{Orientation}(k+1) \cdot \frac{U(k+1)+U(k)}{2}\right]$

Note that the trapezium rule has been used in equation (15) as numerical integration method.

Where:

$$
\begin{aligned}
X & =\left(\begin{array}{c}
x \\
y \\
z \\
\dot{x} \\
\dot{y} \\
\dot{z}
\end{array}\right) \text { are the positions and velocities. } \\
A & =\left(\begin{array}{llllll}
1 & 0 & 0 & \Delta t & 0 & 0 \\
0 & 1 & 0 & 0 & \Delta t & 0 \\
0 & 0 & 1 & 0 & 0 & \Delta t \\
0 & 0 & 0 & 1 & 0 & 0 \\
0 & 0 & 0 & 0 & 1 & 0 \\
0 & 0 & 0 & 0 & 0 & 1
\end{array}\right)=\left(\begin{array}{cc}
I_{3 x 3} & \Delta t \cdot I_{3 x 3} \\
O_{3 x 3} & I_{3 x 3}
\end{array}\right) \\
U & =\left(\begin{array}{l}
a_{u} \\
a_{v} \\
a_{w}
\end{array}\right) \text { are the accelerations. }
\end{aligned}
$$

$$
\begin{aligned}
& B=\left(\begin{array}{c}
\frac{\Delta t^{2}}{2} \cdot I_{3 \times 3} \\
\Delta t \cdot I_{3 \times 3}
\end{array}\right) \\
& \operatorname{Rot}(k+1)=\left(\begin{array}{cc}
I_{3 \times 3} & O_{3 \times 3} \\
O_{3 \times 3} & \text { Rotation }(k+1)
\end{array}\right)
\end{aligned}
$$

where I and $\mathrm{O}$ denote the Identity and Null matrices respectively.

The algorithm to obtain position uses linear acceleration, therefore it is necessary to remove both gravity and centripetal force components from the sensor data to obtain the linear acceleration in a previous step (see Figure 10 and Figure 11).

The Gravity acceleration in the IMU-fixed frame can be calculated as:

$$
\left(\begin{array}{l}
a_{\text {Ugravity }} \\
a_{\text {Vgravity }} \\
a_{\text {Wgravity }}
\end{array}\right)(t+\Delta t)=\text { Rotation }^{-1}(t+\Delta t)\left(\begin{array}{l}
a_{\text {Ugravity }} \\
a_{\text {Vgravity }} \\
a_{\text {Wgravity }}
\end{array}\right)(t)
$$

or using this other representation with the Orientation Matrix:

$$
\left(\begin{array}{l}
a_{\text {Ugravity }} \\
a_{\text {Vgravity }} \\
a_{\text {Wgravity }}
\end{array}\right)(t)=\text { Orientation }^{-1}(t)\left(\begin{array}{l}
0 \\
0 \\
g
\end{array}\right)
$$

where $\mathrm{g}$ denotes the gravity acceleration magnitude.

$\left(\begin{array}{l}0 \\ 0 \\ g\end{array}\right)$ denotes the gravity acceleration vector referenced to the Earth Fixed-Frame and

$\left(\begin{array}{c}a_{\text {Ugravity }} \\ a_{\text {Vgravity }} \\ a_{\text {Wgravity }}\end{array}\right)$ denotes the gravity acceleration vector referenced to the IMU Fixed-Frame



Figure 11. Accelerometer outputs

In general two kinds of rotation can take place in a general movement; the first consists of the rotation of the body around itself and the second consists of the rotation of the body around a point in the space. This is analogous to the movement of the Earth which experiences two rotational movements: One rotation around itself and another one its orbit around the Sun, another typical example would be the general movement of a spinning top.

For this application the first rotational element can be neglected due to its small magnitude, the second however must be taken in to account as follows: 


$$
\left[\begin{array}{l}
a_{\text {ucentripetal }} \\
a_{\text {vcentripetal }} \\
a_{\text {wcentripetal }}
\end{array}\right]=\left[\begin{array}{ccc}
0 & -\omega_{w} & \omega_{v} \\
\omega_{w} & 0 & -\omega_{u} \\
-\omega_{v} & \omega_{u} & 0
\end{array}\right] \cdot\left[\begin{array}{c}
V_{U} \\
V_{V} \\
V_{W}
\end{array}\right]
$$

The centripetal force is given as the cross product of the angular velocity and the linear velocity. This velocity is the linear velocity on the IMU axes obtained in the IMU Fixed-frame, according to the nomenclature followed; this is the velocity in the axes $\mathrm{U}, \mathrm{V}, \mathrm{W}$.

\section{IMU Graphical Interface for Orientation}

As an aid to evaluating the efficiency of the algorithms under development, a graphical tool was generated to show visually the tracking and motion analysis algorithms at work. This consists of a representation of a cube on a PC screen with 6 different colours on each of its sides. As the inertial measurement unit is moved and rotated in real time, this movement is wirelessly transmitted to a base station and the appropriate equations (in MATLAB) implemented on the sensor data. The virtual cube then maps and replicates in real time the movement of the real WIMU which it represents as shown in Figure 12.

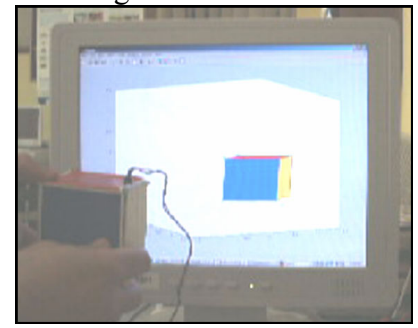

Figure 12. IMU Graphical Interface for Orientation

The cube is defined by eight vertices that form six sides. Figure 13 shows the coordinates of the vertices defining a cube in which the sides are one unit in length:

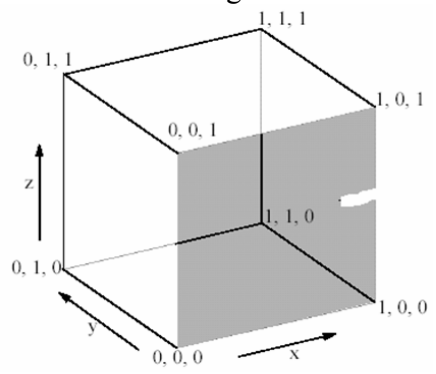

Figure 13. Cube coordinates

The coordinates of all cube vertices can be recalculated after any rotation using the Orientation Matrix and therefore the Graphical interface for orientation consists of collecting the data from the IMU sensors and recalculating the orientation of the real WIMU cube, before redrawing the virtual cube in real time using three different algorithms.

The first algorithm calculates the orientation matrix from the gyroscopes outputs, the second algorithm calculates the orientation matrix from the magnetometers outputs (as a compass) and from the accelerometers outputs (as an inclinometer), and finally the third algorithm calculates the orientation matrix as an output of all IMU sensors using Kalman filter and digital filters.

This results in the cube on the screen mirroring the movement and rotation of the WIMU in real time.

\section{RESULTS}

As anticipated, the orientation estimation using only the gyroscope outputs resulted in a short-term accuracy of inertial measurement (in the order of a few seconds) caused by the drift inherent in these type of sensors, since we were calculating angles by multiplying the output of a gyroscope by time - $t$, any errors in the output of the gyroscope were also multiplied by $t$. This leads to a large error coefficient in the angle calculations.

The described above was experimented using the first algorithm of IMU Graphical Interface for Orientation (as described in section 5).

Orientation estimation from a three axis compass utilising the magnetometer outputs and the data from the accelerometers acting as a tilt sensor resulted in an improved level of accuracy. However the level of precision decreases when the IMU is subjected to rapid movements, or is in presence of ferromagnetic materials or other magnetic fields near the sensor module, as these disturb the local earth magnetic field and, therefore, the orientation estimation. All of this was observed using the second algorithm of IMU Graphical Interface for Orientation (see section 5).

The fusion of the data from the magnetometers, accelerometers and gyroscopes sensors using Kalman Filtering algorithms, along with the use of an appropriate low pass Digital Filter that improves the role of the accelerometers as an inclinometer, eliminated the accuracy decrease discussed above yielding a precise 3D Orientation estimation which was observed using the third algorithm of IMU Graphical Interface for Orientation (see section 5).

As regards tracking of movement, accuracy is an issue due to sensor drift since an IMU calculates position by multiplying the output of an accelerometer by $t^{2}$; any errors in the output of the accelerometer are also multiplied by $\mathrm{t}^{2}$. This leads to huge position errors: in just 60 seconds, a one-dimensional IMU using an accelerometer with an output noise level of just $0.004 \mathrm{~g}$ yields a position uncertainty of about 70 meters. For 3 dimensional tracking, the situation gets worse.

We can see this effect in Figure 14, which is the result of one of the preliminary tests we have carried out. Note that this test was carried out prior to the implementation of the orientation algorithms using the magnetometers and accelerometers outputs as described, and therefore the orientation matrix estimation in this case is poor as a result of being calculated using only sensor data from the gyroscope outputs. This demonstrates the requirement for sophisticated algorithms to map movement using these types of sensors.

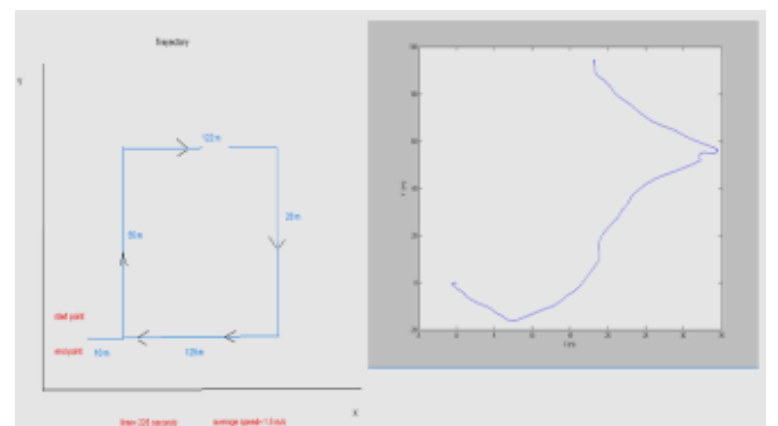

Figure 14. Comparison of a known IMU trajectory versus measured test results 


\section{CONCLUSIONS \& FUTURE WORK}

As far as absolute position tracking is concerned, it has been shown that IMUs cannot be used in isolation for absolute position tracking, however, low cost tracking of movement using inertial MEMS based sensors is conceivable, utilising additional known position input updates as the same manner as the drift in the orientation estimation using gyroscopes was removed using compass and inclinometer updates integrated in the algorithms developed.

A mathematical algorithm for absolute position tracking, which is implemented within a wireless inertial measurement unit (WIMU) based tracking system, has been presented. Future work, will investigate various mechanisms to reduce the drift element associated with typical inertial sensors when used for motion tracking, e.g., a GPS, RFID or triangulation based receiver system to give precise positional updates. These positional updates could then be used along with the mathematical algorithm to provide an accurate 3-Dimensional tracking system.

The accurate results given by the orientation estimation will be the starting point to investigate new algorithms under an approach different from the search of absolute position tracking, the relative position tracking, that is, if we take the Human body as an example of this, one could use an IMUs network with IMUs attached to specific points of the body and use the relative orientation of each other along with the inherent constraints of the human body model (e.g. fixed distance from wrist to elbow and from elbow to shoulder and so on..) to eliminate any integration drift.

\section{ACKNOWLEDGMENTS}

The authors would like to thank all the members of the Ambient Electronic Systems team who have contributed to the project to date. We would like to extend our thanks to Kieran Harney, Analog devices Micromachining Division and Michael Coln, SAR ADC Group Analog Devices, for the provision of devices for this work. We would like to thank the European Commission for funding the MULTIPLEYE project in the IST programme (IST-2001 35108) and the rest of the MULTIPLEYE consortium, the Irish National Arts council for their support, and we would like to acknowledge the support of Enterprise Ireland in funding the D-SYSTEMS project (ATRP-02/227A). Thanks also to Science Foundation Ireland for funding the Tyndall National Access Program (NAP) under which some of this work was carried out.

\section{REFERENCES}

[1] Brendan O'Flynn, Giuseppe Torre, Mikael Fernstrom, Todd Winkler, Andy Lynch, John Barton, Philip Angove, Cian O'Mathuna " Celeritas - A Wearable Sensor System for Interactive Digital Dance Theatre" Proc, BSN2007 - Body Sensor Networks, 4th International Workshop on Wearable and Implantable Body Sensor Networks

[2] G. Welch and G. Bishop, "An Introduction to the Kalman Filter”. (Updated 2004). Department of Computer Science, University of North Carolina.

[3] P. Zhang, J. Gu, E. Milios and Peter Huynh "Navigation with IMU/GPS/Digital Compass with Unscented Kalman Filter". Proceedings of the IEEE International Conference on Mechatronics \& Automation, Niagara Falls, Canada. July 2005. pages $1497-1502,2005$
[4] Dan Simon. "Using non Lineal Kalman Filtering to estimate signals" and "Kalman Filtering". Cleveland State University, Department of Electrical and Computer Engineering.

[5] A Lynch, B Majeed, B O’Flynn, J Barton, F Murphy, K Delaney, S C O'Mathuna "A wireless inertial measurement system (WIMS) for an interactive dance environment", Proc. Sensors \& their Applications XIII, , Greenwich, Kent, England, pp 95-100, September 2005.

[6] Brendan O'Flynn, S. Bellis, K.Mahmood, M. Morris, G. Duffy, K. Delaney, C. O’Mathuna , “A 3-D Miniaturised Programmable Transceiver " Microelectronics International, Volume 22, Number 2, pp. 8-12, 2005.

[7] http://www.tyndall.ie/research/mai-group/index.html

[8] http://www.atmel.com/dyn/products/product_card.asp?part_i $\mathrm{d}=2018$

[9] www.nvlsi.no/index.cfm?obj=product\&act $=$ display\&pro $=64$

[10] http://www.analog.com/UploadedFiles/Data_Sheets/ADXRS 150.pdf

[11] http://www.analog.com/UploadedFiles/Data_Sheets/ADXL2 02E.pdf

[12] http://www.ssec.honeywell.com/magnetic/products.html

[13] http://www.analog.com/en/prod/0\%2C2877\%2CAD7490\%

[14] J. Roberts and P. Corke and J. Cunningham and B. Cook "Experiments in Autonomous Underground Guidance" Proceedings of the 1997 IEEE Intemational Conference on Robotics and Automation, Albuquerque, New Mexico , pp 1898-1903, April 1997.

[15] B. Kemp, A. J. M.W. Janssen, and B. van der Kamp, "Body position can be monitored in 3-D using miniature accelerometers and earth-magnetic field sensors," Electroencephalogr. Clin. Neurophysiol. /Electromyogr.Motor Contr., vol. 109, pp. 484-488, 1998.

[16] Henk J. Luinge and Peter H. Veltink, Member, IEEE "Inclination Measurement of Human Movement Using a 3-D Accelerometer With Autocalibration", IEEE Transactions on Neural Systems and Rehabilitation Engineering, VOL. 12, NO. 1, pp 112-121, MARCH 2004

[17] Daniel Roetenberg, Henk J. Luinge, Chris T. M. Baten, and Peter H. Veltink, Member, IEEE "Compensation of Magnetic Disturbances Improves Inertial and Magnetic Sensing of Human Body Segment Orientation" IEEE Transactions on Neural Systems and Rehabilitation Engineering, VOL 13, NO. 3, pp 395-405, SEPT' 2005

[18] http://www.aisa.uvigo.es/DOCENCIA/AyRobotica//Localiza cion.pdf 